$12-1986$

\title{
Tyrosyl kinase activity is inversely related to prostatic acid phosphatase activity in two human prostate carcinoma cell lines.
}

\author{
Ming-Fong Lin \\ University of Nebraska Medical Center, mlin@unmc.edu \\ Ching-Li Lee \\ Roswell Park Memorial Institute \\ Gail M. Clinton \\ Louisiana State University
}

Tell us how you used this information in this short survey.

Follow this and additional works at: https://digitalcommons.unmc.edu/com_bio_articles

Part of the Medical Biochemistry Commons, and the Medical Molecular Biology Commons

\section{Recommended Citation}

Lin, Ming-Fong; Lee, Ching-Li; and Clinton, Gail M., "Tyrosyl kinase activity is inversely related to prostatic acid phosphatase activity in two human prostate carcinoma cell lines." (1986). Journal Articles:

Biochemistry \& Molecular Biology. 27.

https://digitalcommons.unmc.edu/com_bio_articles/27

This Article is brought to you for free and open access by the Biochemistry \& Molecular Biology at DigitalCommons@UNMC. It has been accepted for inclusion in Journal Articles: Biochemistry \& Molecular Biology by an authorized administrator of DigitalCommons@UNMC. For more information, please contact digitalcommons@unmc.edu. 


\title{
Treatment Response in Depressed Adolescents With and Without Co-Morbid Attention-Deficit/Hyperactivity Disorder in the Treatment for Adolescents with Depression Study
}

\author{
Christopher J. Kratochvil, M.D., Diane E. May, M.S.N., M.A., ${ }^{1}$ Susan G. Silva, Ph.D., ${ }^{2}$ \\ Vishal Madaan, M.D., ${ }^{1}$ Susan E. Puumala, M.S., John F. Curry, Ph.D., ${ }^{2}$ John Walkup, M.D., ${ }^{3}$ \\ Hayden Kepley, Ph.D., ${ }^{4}$ Benedetto Vitiello, M.D., ${ }^{5}$ and John S. March, M.D.?
}

\begin{abstract}
Objective: In the Treatment for Adolescents with Depression Study (TADS), fluoxetine (FLX) and the combination of fluoxetine with cognitive-behavioral therapy $(\mathrm{COMB})$ had superior improvement trajectories compared to pill placebo (PBO), whereas cognitive-behavioral therapy (CBT) was not significantly different from PBO. Because attention-deficit/ hyperactivity disorder (ADHD) and major depressive disorder (MDD) frequently co-exist, we examined whether ADHD moderated these outcomes in TADS.

Method: A total of 439 adolescents with MDD, 12-17 years old, were randomized to FLX, CBT, COMB, or PBO. Random coefficients regression models examined depression improvement in 377 depressed youths without ADHD and 62 with ADHD, including 20 who were treated with a psychostimulant.

Results: Within the ADHD group, the improvement trajectories of the three active treatments were similar, all with rates of improvement greater than PBO. For those without ADHD, only COMB had a rate of improvement that was superior to PBO.

Conclusions: Co-morbid ADHD moderated treatment of MDD. CBT alone or FLX alone may offer benefits similar to COMB in the treatment of MDD in youths with co-morbid MDD and ADHD, whereas monotherapy may not match the benefits of COMB for those without ADHD. The ADHD subgroup analysis presented in this paper is exploratory in nature because of the small number of youths with ADHD in the sample.

Clinical Trial Registry: www.clinicaltrials.gov Identifier: NCT00006286. The TADS protocol and all of the TADS manuals are available on the Internet at https://trialweb.dcri.duke.edu/tads/index.html.
\end{abstract}

\section{Introduction}

A TTENTION-DEFICIT/HYPERACTIVITY DISORDER (ADHD) and depressive disorders are common in the pediatric population, with as many as $20-30 \%$ of children and adolescents diagnosed with ADHD concurrently experiencing a depressive disorder
(Anderson et al. 1987; Biederman et al. 1991; American Psychiatric Association 1994; Angold et al. 1999). Studies by Biederman et al. have demonstrated that adolescents with ADHD have a 2.5 times higher risk for major depressive disorder (MDD) than those without ADHD (Biederman et al. 2008). Because each disorder independently can lead to significant functional impairment, the

\footnotetext{
${ }^{1}$ Department of Psychiatry, University of Nebraska Medical Center, Omaha, Nebraska.

${ }^{2}$ Duke University Medical Center, Durham, North Carolina.

${ }^{3}$ Johns Hopkins University, Baltimore, Maryland.

${ }^{4}$ University of North Carolina at Wilmington, Wilmington, North Carolina.

${ }^{5}$ National Institute of Mental Health, Bethesda, MD.

The statistical consultant for this study was Susan Silva, Ph.D., Duke Clinical Research Institute.

TADS was supported by contract N01 MH80008 from the National Institute of Mental Health to Duke University Medical Center (John S. March, Principal Investigator). Eli Lilly provided fluoxetine and matching placebo under an independent educational grant to Duke University. Eli Lilly had no role in the design or implementation of this study and did not participate in the analysis of the data or in authoring this manuscript. The opinions and assertions contained in this publication are the private views of the authors and are not to be construed as official or as reflecting the views of the National Institute of Mental Health, the National Institutes of Health, or the Department of Health and Human Services.
} 
frequency and impact of simultaneously experiencing the combination of MDD and ADHD is clearly of great clinical concern.

To date, there is a lack of strong evidence supporting a single treatment for co-morbid MDD and ADHD in adolescents. Of the available pharmacological treatments, tricyclic norepinephrine reuptake inhibitors (e.g., desipramine, nortriptyline) have been shown to improve symptoms of ADHD in pediatric populations and depression in adults. The use of these inhibitors in adolescents has been limited by safety and tolerability concerns, and their efficacy as antidepressants has not been well established in this population (Biederman et al. 1993; Wilens et al. 1993; Geller et al. 1999; Prince et al. 2000). Selective serotonin reuptake inhibitors (SSRIs) have demonstrated benefits in adolescent depression and are associated with fewer adverse effects and reduced overdose toxicity, as compared to tricyclic antidepressants, but there is little evidence of efficacy in ADHD (Barrickman 1991; Goldstein and Goodnick 1998; Quintana et al. 2007). Studies have also examined the use of atomoxetine in co-morbid youths. An open-label study of children and adolescents found that atomoxetine, alone or in combination with fluoxetine, improved symptoms of ADHD and depressive symptoms (Kratochvil et al. 2005). However, in a later doubleblind, placebo-controlled study, atomoxetine improved ADHD symptoms, but did not significantly differ from placebo in improving depressive symptoms (Atomoxetine ADHD and Comorbid MDD Study Group et al. 2007). In an open-label trial of bupropion sustained-release, 14 of 24 adolescents were rated as responders in both depression and ADHD, providing data supportive of further placebo-controlled studies of bupropion (Daviss et al. 2001).

Combination pharmacological treatments, such as use of an SSRI along with a stimulant have not been well studied, other than an open-label study using a sample of adults and adolescents with ADHD. This study demonstrated that further global improvements were achieved in attention, behavior, and affect when methylphenidate (MPH) was added to either fluoxetine or sertraline (Findling 1996; Stoll et al. 1996). To date, no large randomized trials have investigated combinational pharmacological treatments of comorbid ADHD and MDD.

Nonpharmacological treatments, such as cognitive-behavioral therapy (CBT) and interpersonal therapy (IPT), have been shown to be moderately effective in the treatment of depressed adolescents (Birmaher et al. 1996; Reinecke et al. 1998; Lewinsohn et al. 1999; Mufson and Sills 2006). Although several studies have suggested that CBT may be helpful in decreasing functional impairment in depressed adolescents, large controlled studies of CBT that are specific to adolescents with co-morbid ADHD and MDD have not been conducted (Antshel and Barkley 2008).

The Treatment for Adolescents with Depression Study (TADS) was a clinical trial of 439 depressed adolescents that compared the effectiveness of four randomized treatments: Pharmacotherapy with fluoxetine (FLX), CBT, the combination of FLX plus CBT $(\mathrm{COMB})$, and clinical management with pill placebo (PBO). Because half of the adolescents in TADS met criteria for at least one co-morbid psychiatric disorder at baseline, the study provided an opportunity to examine the impact that having a co-morbid diagnosis of ADHD had on the outcome of treatment in depressed adolescents who participated in TADS. Given the high level of impairment that may result from ADHD alone, we hypothesized that TADS participants with co-morbid ADHD would be less likely to respond or would respond differentially to TADS treatment across: (1) The 12-week blinded portion of the study, and (2) the entire 36-week treatment period. Our secondary hypotheses are based on past literature of samples of youths with co-morbid ADHD and MDD. We expected that, at baseline, TADS participants with ADHD would have a greater severity of MDD symptoms, a higher level of global impairment, and increased suicidality in comparison to participants without ADHD. Additionally, we expected that TADS participants with ADHD, as compared to those without ADHD, would be more likely to prematurely terminate treatment and drop out of the study.

\section{Methods \\ TADS sample and design}

The rationale, design, methods, and sample characteristics of TADS (TADS 2003; TADS 2005), along with the 12-week acute treatment (TADS 2004) and the overall 36-week treatment outcomes (TADS 2007), have been described in great detail in prior publications. Only those aspects of the study that are directly relevant to these analyses will be presented. In summary, TADS was a publicly funded, randomized controlled trial (RCT) conducted between 2000 and 2003 to evaluate the 12-week and 36-week outcomes of four treatments for MDD: FLX $(n=109)$, CBT $(n=111), \mathrm{COMB}(n=107)$, and $\mathrm{PBO}(n=112)$. PBO was included as a control only during the first 12 weeks of treatment. TADS enrolled 439 adolescents, ages $12-17$ years, including $54.4 \%$ females and $74.8 \%$ Caucasians. Adolescents were excluded if they were considered to be at especially high risk for suicide, as evidenced by a recent history of suicidal behavior or prominent suicidal ideation, or if they had co-morbid substance abuse, bipolar disorder, severe conduct disorder, thought disorder, or were deemed at "high-risk" for self-injurious or aggressive acts (TADS 2003).

After 12 weeks of acute treatment, the two blinded pharmacotherapy treatments (PBO, FLX) were unblinded and PBO treatment was discontinued regardless of response. Youths who were randomized to an active treatment (FLX, CBT, COMB) and had at least partial improvement in symptom severity continued their randomized treatment for 24 additional weeks.

TADS was somewhat unique among pediatric MDD trials in that participants were allowed to continue concomitant treatment with a psychostimulant for ADHD, as long as other eligibility criteria for TADS were met, and the psychostimulant's dose had been stable ( $\leq 25 \%$ change in dose) during the preceding 6 months. The community-prescribing clinician ceded control of the psychostimulant treatment to the TADS pharmacotherapist if the adolescent was assigned to a pharmacological treatment condition, whereas the community clinician continued treatment management if assigned to CBT alone (TADS 2003). The TADS protocol and procedures were approved by each site's local Institutional Review Board, and a National Institute of Mental Health (NIMH) Data and Safety Monitoring Board monitored the study. Written parental consent and written participant assent were obtained for all subjects (TADS 2003; TADS 2005).

The diagnoses of MDD and co-morbid conditions for current and past episodes were established prior to randomization using the Schedule for Affective Disorders and Schizophrenia for SchoolAge Children-Present and Lifetime Version (K-SADS-PL) (Kaufman et al. 1997). Of the 439 enrolled participants in TADS, 60 met current Diagnostic and Statistical Manual of Mental Disorders, $4^{\text {th }}$ edition (DSM-IV) (American Psychiatric Association 1994) criteria for ADHD, of any subtype. Two additional participants were included in our sample that had a history of ADHD and were currently taking a psychostimulant for ADHD, but did not have a current diagnosis of ADHD. For the purpose of this manu- 
script, $62(14.1 \%)$ TADS participants were identified as having ADHD, including 20 participants (32.3\% of the ADHD sample) who had been taking a stable dose of a psychostimulant for ADHD at study entry.

\section{Primary outcome measure}

The primary outcome measure for our analyses was the Children's Depression Rating Scale-Revised (CDRS-R) clinicianbased total scores at baseline evaluation and as reassessed each 6 weeks until the end of acute treatment at week 12 and end of the study treatment at week 36 . The CDRS-R is a 17 -item measure of depression severity over the past week that was administered by an independent evaluator, an experienced clinician who was masked as to the adolescent's assigned treatment. Separate adolescent and parent interviews were completed to yield a clinician summary score, ranging from 17 to 113 , with higher scores representing more severe depression. The scale has good internal consistency (coefficient alpha $=0.85)$, interrater reliability $(r=0.92)$, test-retest reliability $(r=0.78)$, and is correlated with a range of validity indicators, including global ratings and a diagnosis of depression (Poznanski and Mokros 1995). A score of 45 or greater (indicating at least moderate clinical depression) was required for TADS study entry. At TADS baseline, the CDRS-R total scores ranged from 45 to 98 (mean 60, standard deviation $[\mathrm{SD}]=10.4$ ), which translates to a normed $\mathrm{T}$-score of $75.5(\mathrm{SD}=6.43)$, indicating moderate to moderately severe depression (TADS 2005).

\section{Baseline indicators}

Several clinical indicators were used in these analyses to examine differences among the subgroups' characteristics at study entry. Psychometric properties and intercorrelations for these measures are acceptable and have been reported in prior publications (TADS 2003; TADS 2005). The Reynolds Adolescent Depression Scale (RADS) (Reynolds 1987a) is a 30-item adolescent report of depression severity over the past month, with a total score ranging from 30 to 150 . In TADS, more than half $(58.9 \%)$ of participants had a total score of $\geq 77$ at baseline, indicating moderately severe clinical depression, whereas $19.6 \%$ had a total score indicating severe to extreme clinical depression (TADS 2005). Suicidal ideation over the past month was measured by a 15 -item subscale of the Suicidal Ideation Questionnaire-Junior High School Version (SIQ-Jr) (Reynolds 1987b). A cutoff score of $\geq 31$ generally indicates suicidal ideation of sufficient severity to warrant prompt clinical evaluation and is used in this analysis. At baseline, 30.3\% of participants met this flag criterion on the SIQ-Jr. The 100-point Children's Global Assessment Scale (CGAS) (Shaffer et al. 1983) is a clinician rating of general functioning over the past week, with higher scores indicating better functioning.

The Conners' Parent Rating Scale-Revised: Long Version (CPRS-R:L) (Conners 1997) is an 80-item parent rating of ADHD symptoms and other problem behaviors. Total subscale scores are converted into normed T-scores, with 66-70 considered moderately atypical, and $>70$ considered atypical. CPRS-R:L subscale Tscores used in this analysis include: Oppositional, hyperactivity, cognitive problems, ADHD index, global index, and DSM-IV total symptoms, which is further divided into DSM-IV Inattentive symptoms and DSM-IV Hyperactivity/Impulsivity symptoms. The ADHD index is a set of items found useful for identifying "at-risk" youth for ADHD, whereas the Conners global index represents overall psychopathology, loading on two factors: Restless/ impulsivity and emotional lability (Conners 1997).

\section{Premature termination and study dropout}

TADS (2003) defined premature termination as having discontinued assigned study treatment to begin an out-of-protocol treatment, while continuing to participate in study assessments conducted every 6 weeks. The decision to terminate treatment prematurely was based solely upon the clinician's recommendation and not due to mere lack of response or treatment preference. A study dropout was defined as having withdrawn consent or having discontinued assigned treatment and study assessments.

\section{Statistical analyses}

Descriptive statistics were used to summarize sample characteristics. Frequencies and percentages summarized categorical data, whereas means (SD) summarized continuous data. All analyses were conducted using SAS 8.2 (SAS Institute 2002). Due to the exploratory nature of the analysis, statistical significance was set at $p=0.05$ for each nondirectional test. The two ADHD subgroups (MDD with and without ADHD) were compared on key demographic and clinical indicators prior to initiation of treatment. Fisher exact tests were employed for binary measures because some comparisons had small cell sizes, and Student $t$-tests were used for continuous measures. Chi-squared tests were used to test for differences in the proportion of youths randomized to each treatment condition within the two ADHD subgroups.

By definition, a moderator of treatment outcome is a pretreatment variable that is not correlated with treatment, but interacts with treatment resulting in a different profile of treatment effects within the moderator subgroups. A nonspecific predictor of outcome, on the other hand, is a pretreatment variable that is not associated with treatment but significantly influences outcome regardless of treatment condition.

We conducted two intent-to-treat (ITT) analyses on the CDRS-R total scores collected across assessments to determine whether the ADHD diagnosis was a nonspecific predictor or moderator of treatment outcome across 12 weeks of acute treatment and 36 weeks. This analysis was conducted using the methodological approach recommended by Kraemer and colleagues (2002), and was the same analytic approach applied in the primary efficacy analysis of TADS (TADS 2004; TADS 2007), with the exception that the overall analyses incorporated the ADHD subgroup and its interactions tested whether ADHD influenced outcome over 12 weeks and/or 36 weeks.

Longitudinal analyses of the CDRS-R total score were conducted with random coefficients regression models (RRM) (Brown and Prescott 1999) to compare the short-term outcome of four treatments during the initial 12-week acute treatment period and the long-term outcome across 36 weeks of the three active treatments (e.g., COMB, FLX, and CBT). The PBO treatment arm was omitted in the longer-term week-36 analysis due to the discontinuation of PBO after 12 weeks. The ITT analyses included all enrolled participants in the treatment groups to which they were randomly assigned, regardless of their protocol adherence, actual treatment received, and/or subsequent withdrawal or deviation from the protocol. The overall RRM model for the week-12 and week-36 analyses included the: (1) Fixed effects of site, treatment, time, treatment-by-time, ADHD subgroup, and its two- and three-way interactions, and (2) random effects of participant and participantby-time on the CDRS-R total score. Site was retained in the model as a covariate because treatments were nested within site and time was defined as the natural log of time +1 (measured as days since randomization). Although the temporal effects were significantly 
linear over the initial 12 weeks, the relationship was quadratic (nonlinear) over the 36 weeks. Thus, the final analysis model for the initial 12 weeks was a hierarchical linear mixed model, whereas the week-36 data was analyzed using a hierarchical quadratic mixed model.

For the 439 depressed adolescents included in the week-12 analysis, the two subgroups included youths diagnosed with ADHD $(n=62)$ and without ADHD $(n=377)$. Because the PBO arm was omitted from the week-36 analysis, the sample of 327 included a smaller number of youths with ADHD $(n=43)$ and without ADHD $(n=284)$. If the above omnibus model indicated a significant ADHD-by-treatment or ADHD-by-treatment-by-time interaction, then a separate RRM for each ADHD subgroup was conducted to examine the fixed effects of site, treatment, treatment-by-time, as well the random effects of participant and participant-by-time. For each conducted RRM that resulted in a significant treatment or treatment-by-time effect $(p \leq 0.05)$, a posteriori paired contrasts were conducted to examine treatment differences.

For the 62 youths in the ADHD subgroup, we also conducted a $2 \times 4$ analysis of variance using a general linear model approach to examine the effects of stimulant medication use and treatment assignment and interaction on CDRS-R total scores at baseline. For this analysis, we included the subgroup of depressed adolescents with ADHD who, at baseline, were currently treated with a stimulant medication $(n=20)$ and those untreated for $\operatorname{ADHD}(n=42)$.

\section{Results}

Of the 439 enrolled adolescent participants in TADS, $62(14.1 \%)$ were assessed as having a diagnosis of ADHD at study entry. TADS participants with ADHD had a mean age of 14.6 (SD 1.5) years at time of consent. There were twice as many males $(66.1 \%)$ as females $(p<0.004)$ in our sample of depressed adolescents who had a diagnosis of co-morbid ADHD. No difference was found in assigned treatment among participants who did or did not have a diagnosis of $\operatorname{ADHD}\left(\chi^{2}=1.10, p=0.777\right)$. Among the 62 youths with ADHD, 14 (22.6\%) were assigned to CBT, 14 (22.6\%) assigned to FLX, 15 (24.2\%) to COMB, and 19 (30.6\%) to PBO. Table 1 presents baseline demographic and clinical indicators for the subgroups of TADS participants who did or did not have a comorbid diagnosis of ADHD at study entry and who were included in the week-12 analysis.

Mean (SD) for T-scores on the CPRS-R:L subscales, measuring parent-reported ADHD symptoms and other problem behaviors, are presented in Table 2.

\section{Premature termination and study dropout}

There was no statistical difference $(p=0.999)$ among participants with or without a diagnosis of ADHD who prematurely terminated their assigned treatment by week 12, after a TADS clinician recommended an out-of-protocol treatment, including 6 (9.7\%) with ADHD and 36 (9.6\%) without ADHD. Furthermore, there was no significant difference found in the rate of study dropout at end of the 12-week acute treatment $(p=0.378)$, including $9(14.5 \%)$ with ADHD and 39 (10.3\%) without ADHD. Additionally, there was no significant difference $(p=0.755)$ found in the rate of dropout for the overall 36-week treatment among participants with or without a diagnosis of ADHD, including 17 (27.4\%) youths with ADHD and 96 (25.5\%) without ADHD who dropped out of TADS.

\section{2-Week blinded treatment outcome $(\mathrm{N}=439)$}

The overall linear RRM completed for the 439 enrolled participants over the 12-week acute blinded-treatment period indicated a significant site effect $\left(\mathrm{F}_{(12,422)}=1.78 ; p=0.050\right)$, linear time effect $\left(\mathrm{F}_{(1,422)}=457.92 ; p<0.001\right)$, treatment-by-time interaction $\left(\mathrm{F}_{(3,421)}=3.38 ; p=0.018\right)$, and ADHD-by-treatment-by-time interaction $\left(\mathrm{F}_{(3,421}=3.11 ; p=0.026\right)$, with the remaining fixed effects nonsignificant $(p>0.050)$. The significant ADHD interaction demonstrated that improvement in depression was moderated by ADHD status prior to treatment or having a diagnosis of ADHD prior to study entry. A separate RRM was then completed for each ADHD subgroup and showed that each subgroup had a significant time (without ADHD: $\mathrm{F}_{(5,355)}=945.58 ; p \leq 0.001$; with ADHD: $\left.\mathrm{F}_{(1,53)}=145.18 ; p<0.001\right)$ and treatment-by-time interaction (without ADHD: $\mathrm{F}_{(3,355)}=9.55 ; p<0.001$; $\operatorname{ADHD} \mathrm{F}_{(3,53)}=3.03$; $p=0.038$ ) effects.

Table 1. Baseline Indicators of Depressed Adolescents with and without ADHD

\begin{tabular}{|c|c|c|c|c|}
\hline & & TADS youth with $A D H D \mathrm{n}=62$ & Without ADHD $\mathrm{n}=377$ & $\mathrm{p}$ value \\
\hline Gender (male) & $n(\%)$ & $41(66.13 \%)$ & $159(42.18 \%)$ & $<0.005$ \\
\hline Caucasian & $n(\%)$ & $50(80.65 \%)$ & $274(72.68 \%)$ & 0.214 \\
\hline Initial depressive episode & $n(\%)$ & $50(81.97 \%)$ & $319(86.68 \%)$ & 0.322 \\
\hline Depression severity (CDRS-R total) & Mean (SD) & $57.58(9.87)$ & $60.52(10.43)$ & 0.030 \\
\hline Adolescent-reported MDD (RADS total) & Mean (SD) & $74.46(12.61)$ & $80.01(14.48)$ & 0.003 \\
\hline Global functioning (CGAS rating) & Mean (SD) & $50.05(7.70)$ & $49.57(07.44)$ & 0.690 \\
\hline Potential risk of suicidality flag (SIQ-Jr) & $n(\%)$ & $14(23.73 \%)$ & $111(30.08 \%)$ & 0.358 \\
\hline Current dysthymia disorder & $n(\%)$ & $11(17.74 \%)$ & $35(9.31 \%)$ & 0.070 \\
\hline Current anxiety disorder & $n(\%)$ & $12(19.35 \%)$ & $108(28.72 \%)$ & 0.166 \\
\hline Current oppositional defiant disorder & $n(\%)$ & $18(29.03 \%)$ & $40(10.61 \%)$ & $<0.001$ \\
\hline Current substance abuse disorder & $n(\%)$ & $1(1.61 \%)$ & $6(1.59 \%)$ & 1.000 \\
\hline Current alcohol abuse disorder & $n(\%)$ & $1(1.61 \%)$ & $2(0.53 \%)$ & 0.358 \\
\hline
\end{tabular}

Table's values are expressed as $n=$ number of randomized participants (percentage [\%]), or as a group mean (standard deviation [SD]). For mean data, $p$ values represent the Fisher exact tests for binary measures and Student $t$-tests for continuous measures.

Anxiety disorder includes the diagnoses of panic disorder, separation anxiety disorder, specific phobia, social phobia, agoraphobia, generalized anxiety disorder, posttraumatic stress disorder, and/or acute stress disorder.

Abbreviations: $\mathrm{ADHD}=$ Attention-deficit/hyperactivity disorder; TADS $=$ Treatment for Adolescents with Depression Study; $C D R S-R=$ Children's Depression Rating Scale-Revised; MDD = major depressive disorder; RADS = Reynolds Adolescent Depression Scale; SIQ-Jr=Suicidal Inventory Questionnaire-Junior High School Version. 
Table 2. Conners' Parent Rating Scale-Revised Subscale T-Scores

\begin{tabular}{|c|c|c|c|c|}
\hline & Youth With ADHD $\mathrm{n}=62$ & Without ADHD $\mathrm{n}=377$ & $\mathrm{t}-$ Value & $\mathrm{p}$ Value \\
\hline Oppositional subscale & $74.62(10.89)$ & $70.05(13.25)$ & -2.87 & 0.005 \\
\hline Cognitive problems subscale & $76.35(09.25)$ & $67.57(11.95)$ & -4.51 & $<0.001$ \\
\hline Hyperactivity subscale & $77.41(15.56)$ & $62.55(14.91)$ & -5.44 & $<0.001$ \\
\hline ADHD index & $78.57(08.71)$ & $67.81(11.68)$ & -7.25 & $<0.001$ \\
\hline Conners' Global Index & $80.23(11.38)$ & $71.01(13.03)$ & -4.51 & $<0.001$ \\
\hline DSM-IV Total Symptoms & $80.54(9.99)$ & $67.33(12.37)$ & -7.88 & $<0.001$ \\
\hline DSM-IV Inattention Symptoms & $78.40(08.88)$ & $68.20(12.25)$ & -6.73 & $<0.001$ \\
\hline DSM-IV Hyperactivity/Impulsivity & $77.06(15.62)$ & $60.89(13.35)$ & -6.01 & $<0.001$ \\
\hline
\end{tabular}

Conners' Parent Rating Scale, Revised-Long Version subscale values are expressed as T-score group means and standard deviation (SD) at baseline. $p$ values are for Student $t$-tests. ADHD Index subscale consists of items useful for identifying "at risk" youth for ADHD. Global Index measures psychopathology of two factors: Restless/impulsivity and emotional lability. DSM-IV Total Symptoms subscale items match to diagnostic criteria of combined subtype of ADHD, while DSM-IV Inattention Symptoms subscale matches to inattentive subtype of ADHD, and DSM-IV Hyperactivity/Impulsivity subscale matches to ADHD subtype.

Abbreviations: ADHD = Attention-deficit/hyperactivity disorder; DSM-IV = Diagnostic and Statistical Manual of Mental Disorders, $4^{\text {th }}$ edition.

For the 377 depressed youths in TADS without ADHD, the profile for the rate of improvement across 12 weeks was: $\mathrm{COMB}>$ $(\mathrm{FLX}>\mathrm{CBT})=\mathrm{PBO}$. The COMB treatment condition had a significantly faster average trajectory of change across 12 weeks $(p<0.009)$ relative to FLX, CBT, or PBO. Neither FLX $(p=0.425)$ nor CBT $(p=0.065)$ was significantly different than $\mathrm{PBO}$ in terms of rate of improvement, although FLX $(p=0.008)$ had a significantly greater average improvement rate compared to CBT, indicating that CBT had the most gradual improvement trajectory among the active treatments.

A different pattern of treatment effects was observed for the 62 depressed youths with ADHD, which was $(\mathrm{CBT}=\mathrm{FLX}=\mathrm{COMB})$ $>$ PBO. Compared to $\mathrm{PBO}$, the treatment conditions of $\mathrm{CBT}$ $(p=0.013), \operatorname{FLX}(p=0.024)$, and COMB $(p=0.046)$ showed significantly greater average rates of improvement during the 12week blinded treatment. None of the three active treatments was significantly different in terms of their change trajectories (all $p>0.050)$.

Improvement, as measured by lower mean CDRS-R scores, was observed in all four treatment conditions of both subgroups over the 12-week blinded-treatment period, which included three assessment visits at baseline and at weeks 6 and 12. The mean CDRS-R scores over the 12-week period, adjusted for the fixed and random effects in the analytic model, are presented in Table 3 for the two ADHD subgroups, and presented graphically in Fig. 1.

The CGI-I response rate at week 12, using last observation carried forward (LOCF) when a score was missing, was $53.3 \%$ for the youths without ADHD and $45.2 \%$ for those with ADHD $(\mathrm{chi}=1.4$, degrees of freedom $[\mathrm{df}]=1, p=0.230$, effect size $=$ 0.2 ). Table 4 presents the between-treatment effect sizes at week 12 for the two subgroups of depressed adolescents, with and without ADHD.

\section{6-week treatment outcome $(\mathrm{N}=327)$}

The analysis, which included an adjustment for a significant quadratic temporal relationship $\left(\mathrm{time}^{2}\right)$ of the three active treatment conditions and the CDRS-R total scores across 36 weeks, also demonstrated a moderator effect for ADHD status. The overall analysis demonstrated a significant time $\left(\mathrm{F}_{(1,1456)}=45.59 ; p<\right.$ 0.001), ADHD-by-time ${ }^{2}$ (quadratic) interaction $\left(\mathrm{F}_{(1,1298}=5.04\right.$; $p=0.025)$, ADHD-by-treatment-by time interaction $\left(\mathrm{F}_{(2,145)}=\right.$ $5.60 ; p=0.004)$, and ADHD-by-treatment-by-time ${ }^{2}$ interaction $\left(\mathrm{F}_{(2,1297}=5.53 ; p=0.004\right)$, while the remaining fixed effects were nonsignificant (all $p<0.050$ ). The significant ADHD-by-treatment-by-time ${ }^{2}$ interaction demonstrated that having a diagnosis of ADHD had a moderating effect during the 36-week treatment period.

For the 284 youths without ADHD, there was a significant treatment-by-time ${ }^{2}$ interaction $\left(\mathrm{F}_{(2,1127)}=23.56 ; p<0.001\right)$ in which there was a significant difference in the improvement trajectory of this subgroup. As during the initial 12 weeks, the most rapid rate of improvement was observed in the COMB condition (all $p>0.050$ ), which was significantly different from CBT and FLX in terms of trajectories, with CBT having a significantly more gradual improvement over the 36 weeks relative to COMB and FLX. Paired

Table 3. Mean Adjusted CDRS-R Total Scores for the 12-Week Analysis $(\mathrm{N}=439)$

\begin{tabular}{|c|c|c|c|c|c|c|c|c|}
\hline & \multicolumn{2}{|c|}{ Fluoxetine alone } & \multicolumn{2}{|c|}{ CBT alone } & \multicolumn{2}{|c|}{ Combination $(C B T+$ fluoxetine $)$} & \multicolumn{2}{|c|}{ Pill placebo } \\
\hline & $\begin{array}{l}\text { Youth with } \\
\text { ADHD } \\
\mathrm{n}=14\end{array}$ & $\begin{array}{c}\text { Without } \\
\text { ADHD } \\
\mathrm{n}=95\end{array}$ & $\begin{array}{c}\text { Youth with } \\
\text { ADHD } \\
\mathrm{n}=14\end{array}$ & $\begin{array}{l}\text { Without } \\
\text { ADHD } \\
\mathrm{n}=97\end{array}$ & $\begin{array}{l}\text { Youth with } \\
\text { ADHD } \\
\mathrm{n}=15\end{array}$ & $\begin{array}{l}\text { Without } \\
\text { ADHD } \\
\mathrm{n}=92\end{array}$ & $\begin{array}{l}\text { Youth with } \\
\text { ADHD } \\
\mathrm{n}=19\end{array}$ & $\begin{array}{l}\text { Without } \\
\text { ADHD } \\
\mathrm{n}=93\end{array}$ \\
\hline Base & $56.08 \pm 3.93$ & $59.36 \pm 4.24$ & $0.01 \pm 4.66$ & 4.78 & $0+7$ & 61.14 & \pm 4.50 & 62.3 \\
\hline Week 6 & $37.05 \pm 6.03$ & $40.37 \pm 7.33$ & $39.45 \pm 5.68$ & $44.98 \pm 8.22$ & $40.73 \pm 8.54$ & $37.58 \pm 7.67$ & $45.25 \pm 5.30$ & $44.59 \pm 7.42$ \\
\hline Week 12 & $33.29 \pm 6.42$ & $36.83 \pm 8.11$ & $35.95 \pm 6.34$ & $42.35 \pm 8.96$ & $37.08 \pm 9.42$ & $33.30 \pm 8.14$ & $43.31 \pm 5.65$ & $41.39 \pm 8.07$ \\
\hline
\end{tabular}

Mean adjusted scores derived from the subgroup analyses.

Abbreviations: CDRS-R = Children's Depression Rating Scale-Revised; ADHD = attention-deficit/hyperactivity disorder; CBT $=$ cognitive-behavioral therapy. 

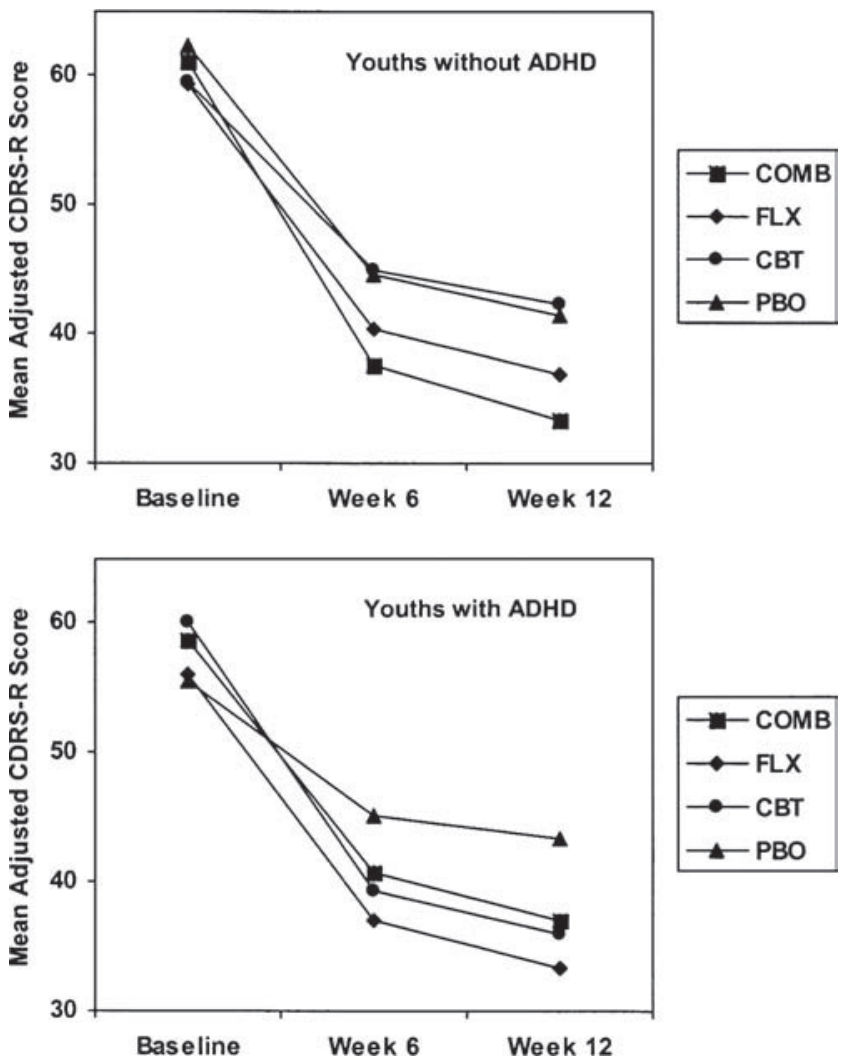

FIG. 1. CDRS-R adjusted total scores for youths with and without ADHD across the 12-week analysis. Shown is the profile of treatment-group effects based on the hierarchical linear mixed regression models completed to compare the outcome of four treatments during the initial 12 weeks. CDRS-R $=$ Children's Depression Rating Scale-Revised; ADHD = attention-deficit $/$ hyperactivity disorder; $\mathrm{COMB}=$ combined treatment of cognitive-behavioral therapy and fluoxetine; FLX = fluoxetine; $\mathrm{CBT}=$ cognitive-behavioral therapy; $\mathrm{PBO}=$ pill placebo.

contrasts of mean CDRS-R scores were conducted at week 36 to test for between-treatment differences within each ADHD subgroup. No significant difference was found among any of the active treatment groups in terms of their mean CDRS-R scores.

The ADHD subgroup $(n=43)$ analysis did not demonstrate any significant treatment-by-time ${ }^{2}$ or treatment-by-time interactions. To examine between-treatment differences in light of the small sample size per treatment condition, exploratory paired contrasts were conducted in the absence of a significant treatment-by-time ${ }^{2}$ interaction within this subgroup. As expected, there were no significant differences in change trajectories or in CDRS-R total scores at week 36 (all $p>0.050$ ). The separate RRM analysis for each ADHD subgroup revealed the following, as depicted in Fig. 2. CGI-I response rate at week 36, using LOCF when a score was missing, was $75.4 \%$ for the youths without ADHD and $62.8 \%$ with ADHD (chi $=3.0, \mathrm{df}=1, p=0.080)$.

\section{Psychostimulant treatment of $A D H D$}

At time of randomization to TADS treatment, 20 (32.2\%) of the 62 depressed adolescents who were diagnosed with ADHD were taking a stimulant medication to treat ADHD. Our analysis showed that CDRS-R depression scores did not prove to be significantly different for the group of 20 youths treated with a psychostimulant,
Table 4. Between-Treatment Effect Sizes at Week 12 FOR ADOLESCENTS, WITH AND WITHOUT ADHD

\begin{tabular}{lcc}
\hline Treatment comparison & $\begin{array}{c}\text { Youths } \\
\text { without ADHD }\end{array}$ & $\begin{array}{c}\text { Youths } \\
\text { with ADHD }\end{array}$ \\
\hline Active treatment versus PBO & & \\
COMB versus PBO & 1.0 & 0.8 \\
FLX versus PBO & 0.6 & 1.7 \\
CBT versus PBO & -0.1 & 1.2 \\
\hline
\end{tabular}

Abbreviations: $\mathrm{ADHD}=$ Attention-deficit/hyperactivity disorder; $\mathrm{PBO}=$ pill placebo; $\mathrm{COMB}=$ combinational treatment with cognitive-behavioral therapy plus fluoxetine; FLX = fluoxetine; CBT = cognitive-behavioral therapy.

as compared to the group of 43 youths who were not treated for $\operatorname{ADHD}\left(\mathrm{F}_{(1,54)}=0.34, p=0.056\right)$. Additionally, no significant treatment-by-psychostimulant use interaction was found $(p>0.050)$.

\section{Discussion}

This analysis examines the impact of having a co-morbid diagnosis of ADHD on the outcome of treatment for depression in
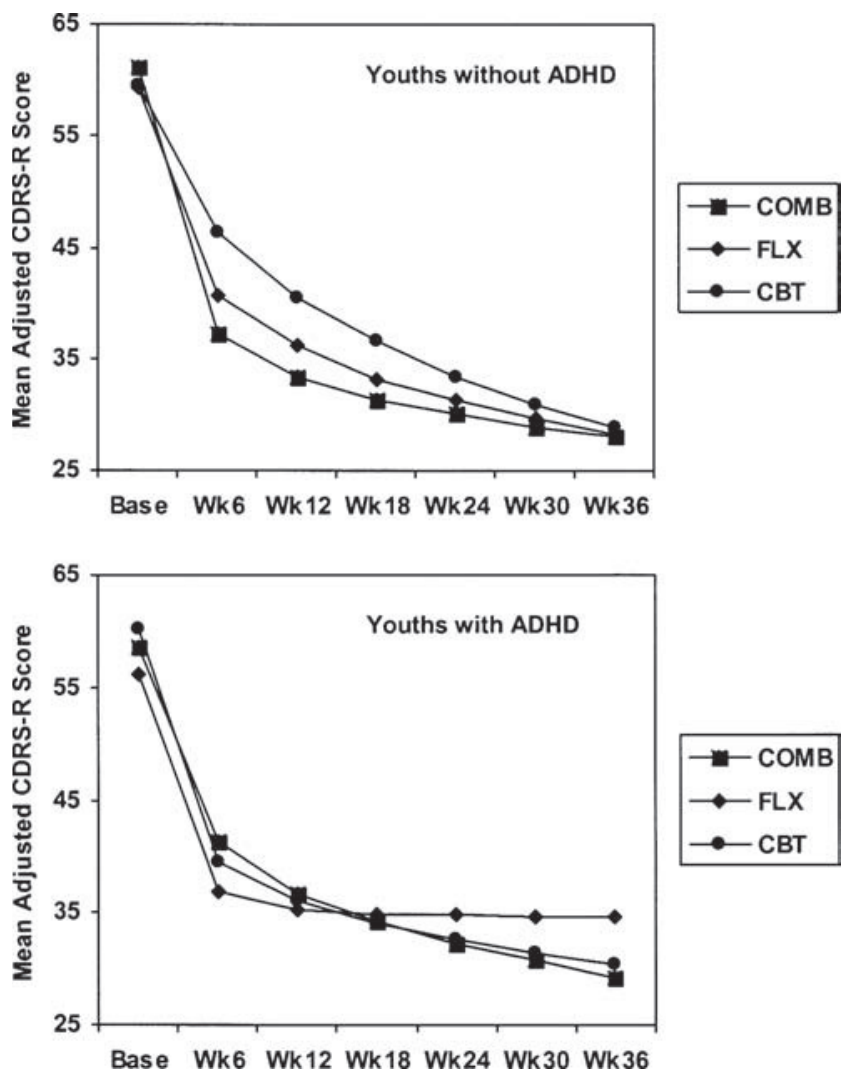

FIG. 2. CDRS-R adjusted total scores for youths with and without ADHD across the 36-week analysis. Shown is the profile of treatment-group effects based on the random coefficients regression models completed to compare the outcome of three treatments across 36 weeks. CDRS-R = Children's Depression Rating Scale-Revised; ADHD = attention-deficit/hyperactivity disorder; $\mathrm{COMB}=$ combined treatment of cognitive-behavioral therapy and fluoxetine; FLX = fluoxetine; $\mathrm{CBT}=$ cognitive behavioral therapy. 
adolescents who participated in TADS. Approximately one in seven $(14.1 \%)$ adolescent participants met criteria for a diagnosis of ADHD. As hypothesized, ADHD was a moderator of treatment response. We found a differential response to TADS treatment in the depressed youths with a co-morbid diagnosis of ADHD, as compared to without ADHD. The ADHD subgroup analyses presented in this paper are exploratory in nature, due to the limited number of youths in TADS with ADHD, which affects the statistical power to detect significant differences between the subgroups and increases the possibility of Type II errors (i.e., false negatives).

Although our expectation that depressed adolescent participants with ADHD would have a less robust response to treatment than those without ADHD was not met, our prediction of a differential response moderated by having a diagnosis of ADHD was supported. In TADS youths who had ADHD, the three treatment groups of COMB, FLX, and CBT had a similarly greater average rate of improvement during the 12-week blinded treatment compared to PBO. This rate of improvement similarly continued during the remaining 24 weeks of the study for all three active treatments. This is a different trajectory, based on rate of improvement, compared to the group of depressed adolescents in TADS who did not have ADHD.

In our analysis of youths without ADHD, the COMB treatment group experienced the greatest rate of improvement during the initial 12 weeks, with COMB superior to PBO. CBT continued to have a more gradual rate of improvement over the 36-week period compared to COMB or FLX. By end of treatment at week 36, all active treatments were similarly effective in alleviating depressive symptoms in TADS youths, regardless of whether or not they had ADHD.

Our secondary hypothesis - that participants with ADHD would prematurely terminate their assigned treatment and drop out of the study more often than participants without ADHD—was not supported. We found no significant difference between the youths with or without ADHD in the rate of study dropout or premature termination (i.e., study treatment stopped due to starting a clinicianrecommended nonprotocol treatment). Finally, our hypothesis that ADHD youths would have higher global impairment and higher level of suicidality compared to youths without ADHD was also not supported. Instead we found that as a group ADHD youths did not differ from those without ADHD in their overall global impairment or their level of suicidality. In fact, althought we predicted a higher severity of MDD in the ADHD youths, we found that ADHD youths in the TADS sample had significantly less severe depressive symptoms at baseline than those without ADHD, on both the clinician-administered CDRS-R and on the adolescent-completed RADS. Additionally, there was no difference between those adolescents with and those without ADHD in the age of onset of MDD, duration of MDD episode, or rate of recurrent depression (all $p>0.050)$. There was, however, an increased rate of concurrent dysthymia in those with ADHD (17.7\% vs. 9.3\%; $p=0.0448)$.

We speculate regarding possible reasons for these differing results, particularly that CBT had a superior trajectory of improvement over PBO in youths with ADHD, but not for those without ADHD. We initially believed that depressed youths with ADHD would benefit less from CBT due to the cognitive deficits of ADHD and the cognitive requirements in $\mathrm{CBT}$, such as completing and applying homework-based concepts. In TADS, parents of ADHD youths typically described their teens as experiencing significant levels of inattention, hyperactivity, impulsivity, and emotional lability, in addition to their depression. Despite the elevated levels of ADHD symptoms, CBT, as offered in TADS, provided an inter- vention that was particularly beneficial for the depressed adolescents with ADHD. It is possible that the CBT in TADS overlaps with interventions having demonstrated efficacy in the treatment of ADHD in children (Birmaher et al. 1996; Lewinsohn et al. 1999; MTA Cooperative Group 1999), such as goal setting, problem solving, charting of affect regulation, and contingency management. Unfortunately, data on the role of CBT per se in the treatment of ADHD in children and adolescents are more limited than other psychosocial interventions, with seminal studies like the MTA focusing on behavioral interventions rather than CBT.

As the largest study of adolescent depression completed to date, TADS is one of the few pediatric depression studies with a design and size adequate to investigate beyond primary comparative outcomes. Unfortunately, our exploratory analysis of the 62 youths in TADS who had a co-morbid diagnosis of ADHD is still quite limited in size, which limits the generalizability of our results. If replicated in future studies, our findings could strengthen guidance in the treatment of adolescents with co-morbid MDD and ADHD. In particular, our findings would suggest that any of the three TADS interventions of CBT, FLX, or COMB may be an appropriate initial treatment for depression in an adolescent with co-morbid depression and ADHD. The treatment recommendations must be individualized, however, taking into consideration other possible clinical concerns, co-morbidities, treatment availability, and family preference,

All three active treatment groups had an average decrease in mean CDRS-R score of greater than 20 points at week 12, yet slightly less than half of the co-morbid youths reached much or very much improvement after 3 months of treatment, suggesting the need for better treatments for depression, particularly with comorbid ADHD. Further examination of the role of concomitant ADHD pharmacotherapy during treatment of adolescent depression might prove clinically invaluable, but this is well beyond the feasibility of our subsample of 20 youths who were treated with a stimulant medication. We recommend conducting larger studies that are more adequately powered to examine moderator and mediator analyses, which are needed to better guide clinical treatment of common co-morbidities in adolescents.

TADS was unique among pediatric MDD trials in allowing depressed adolescents with co-morbidities such as ADHD to continue taking daily treatment with a psychostimulant. By allowing such real-world aspects in a controlled study, data such as this become increasingly relevant to clinical practice. In our analyses, ADHD moderated the short- and longer-term effects of MDD treatment in youths with co-morbid ADHD compared to without ADHD. Our findings indicated that CBT, COMB, and FLX had similar improvement trajectories in depressed youths with ADHD, whereas COMB had superior improvement in youths without ADHD. Thus, our results suggest that any of these three TADS interventions of CBT, FLX, or COMB may be an appropriate initial treatment of depression in adolescents with co-morbid ADHD.

\section{Disclosures}

Dr. Kratochvil has or does receive grant support from Eli Lilly, Ortho-McNeil, Shire, Abbott, Pfizer, Somerset, Cephalon, and NIMH; he has been a consultant for Eli Lilly, AstraZeneca, Abbott, and Pfizer; and he is currently receiving medication at no cost from Eli Lilly for a study. Ms. May has received grant support from Shire, Abbott, Somerset, Cephalon, and NIMH. Dr. Silva is a consultant to Pfizer. Dr. March owns stock in MedAvante and has been a consultant for Pfizer, Wyeth, Eli Lilly, and GlaxoSmithKline; 
he has received research support from Eli Lilly and has served on data safety monitoring boards for AstraZeneca and Johnson \& Johnson; he is currently receiving medication at no cost from Pfizer and Eli Lilly for a study; and he has received royalty payments for the Multidimensional Anxiety Scale for Children. Dr. Walkup reports receiving consulting fees from Eli Lilly and Jazz Pharmaceuticals and fees for legal consultation to defense counsel and submission of written reports in litigation involving GlaxoSmithKline, receiving lecture fees from CMP Media, Medical Education Reviews, McMahon Group, and DiMedix, and receiving support in the form of free medication and matching placebo from Eli Lilly and free medication from Abbott for clinical trials funded by the NIMH. All other authors have no financial ties or conflicts of interest to report. Eli Lilly provided fluoxetine and matching placebo under an independent educational grant to Duke University.

\section{Acknowledgments}

The authors would like to thank the members of the Treatment for Adolescents with Depression Study (TADS) Team. The TADS is coordinated by the Department of Psychiatry and Behavioral Sciences and the Duke Clinical Research Institute at Duke University Medical Center, Durham, North Carolina, in collaboration with the NIMH. The Coordinating Center principal collaborators are John S. March, Susan Silva, Stephen Petrycki, John Curry, Karen Wells, John Fairbank, Barbara Burns, Marisa Domino, and Steven McNulty. The NIMH principal collaborators are Benedetto Vitiello and Joanne Severe. Principal investigators and coinvestigators from the clinical sites are as follows: Charles Casat, Jeanette Kolker, and Karyn Riedal (Carolinas Medical Center, Charlotte, North Carolina); Norah Feeny, Robert Findling, Sheridan Stull, and Susan Baab (Case Western Reserve University, Cleveland, Ohio); Elizabeth B. Weller, Michele Robins, Ronald A. Weller, and Naushad Jessani (The Children's Hospital of Philadelphia, Philadelphia, Pennsylvania); Bruce Waslick (Baystate Health/Tufts University, Springfield, Massachusetts), Michael Sweeney, and Randi Dublin (Columbia University, New York, New York); John Walkup, Golda Ginsburg, Elizabeth Kastelic, and Hyung Koo (The Johns Hopkins University, Baltimore, Maryland); Christopher Kratochvil, Diane May, Randy LaGrone, and Brigette Vaughan (University of Nebraska, Omaha); Anne Marie Albano (Columbia University), Glenn S. Hirsch, and Elizabeth Podniesinki (New York University, New York); Mark Reinecke, Bennett Leventhal, Gregory Rogers, and Rachel Jacobs (University of Chicago, Chicago, Illinois, and Northwestern University, Evanston, Illinois); Sanjeev Pathak, Jennifer Wells, Sarah Arszman, and Arman Danielyan (Cincinnati Children's Hospital Medical Center, Cincinnati, Ohio); Paul Rohde, Anne Simons, James Grimm, and Stephenie Frank (University of Oregon, Eugene); Graham Emslie, Beth Kennard, Carroll Hughes, and Taryn L. Mayes (The University of Texas Southwestern Medical Center, Dallas); David Rosenberg, Nili Benazon, Michael Butkus, and Marla Bartoi (Wayne State University, Detroit, Michigan); and Kelly Posner, for the Columbia University Suicidality Classification Group. James Rochon (Duke Clinical Research Institute, Durham) was statistical consultant for TADS.

\section{References}

American Psychiatric Association: Diagnostic and Statistical Manual of Mental Disorders, $4^{\text {th }}$ edition. (DSM-IV). Washington (DC): American Psychiatric Association, 1994.
Anderson JC, Williams S, McGee R, Silva PA: DSM-III disorders in preadolescent children: Prevalence in a large sample from the general population. Arch Gen Psychiatry 44:69-76, 1987.

Angold A, Costello J, Erkanli A: Comorbidity. J Child Psychol Psychiatry 40:57-87, 1999.

Antshel KM, Barkley R: Psychosocial interventions in attention deficit hyperactivity disorder. Child Adolesc Psychiatr Clin N Am 17:421-437, 2008.

Atomoxetine ADHD and Comorbid MDD Study Group: Bangs ME, Emslie GE, Spencer TJ, Ramsey JL, Carlson C, Bartky EJ, Busner J, Duesenberg DA, Harshawat P, Kaplan SL, Quintana H, Allen AJ, Sumner CR: Efficacy and safety of atomoxetine in adolescents with ADHD and major depression. J Child Adolesc Psychopharmacol 17:407-420, 2007.

Barrickman L, Noyes R, Kuperman S, Schumacher E, Verda M: Treatment of ADHD with fluoxetine: A preliminary trial. J Am Acad Child Adolesc Psychiatry 30:762-767, 1991.

Biederman J, Newcorn J, Sprich S: Comorbidity of attention deficit hyperactivity disorder with conduct, depressive, anxiety, and other disorders. Am J Psychiatry 148:564-577, 1991.

Biederman J, Baldessarini RJ, Wright V, Keenan K, Faraone S: A double-blind placebo controlled study of desipramine in the treatment of ADD: III. Lack of impact of comorbidity and family history factors on clinical response. J Am Acad Child Adolesc Psychiatry 32:199-204, 1993.

Biederman J, Ball S, Monuteaux M, Mick E, Spencer T, McCreary M, Cote M, Farone S: New insights into the comorbidity between ADHD and major depression in adolescent and young adult females. J Am Acad Child Adolesc Psychiatry 47:426-434, 2008.

Birmaher B, Ryan ND, Williamson DE, Brent DA, Kaufman J, Dahl RE, Perel J, Nelson B: Childhood and adolescent depression: A review of the past 10 years, Part I. J Am Acad Child Adolesc Psychiatry 35:1427-1439, 1996.

Conners CK: Technical Manual for the Conners' Rating ScalesRevised. North Tonawanda (New York): Multi-Health Systems, 1997.

Daviss WB, Bentivoglio P, Racusin R, Brown KM, Bostic JQ, Wiley L: Bupropion sustained release in adolescents with comorbid attention-deficit/hyperactivity disorder and depression. J Am Acad Child Adolesc Psychiatry 40:307-314, 2001.

Findling RL: Open-label treatment of comorbid depression and attentional disorders with co-administration of serotonin reuptake inhibitors and psychostimulants in children, adolescents, and adults: A case series. J Child Adolesc Psychopharmacol 6:165-175, 1996.

Geller B, Reising D, Leonard HL, Riddle MA, Walsh BT: Critical review of tricyclic antidepressant use in children and adolescents. J Am Acad Child Adolesc Psychiatry 38:513-516, 1999.

Goldstein BJ, Goodnick PJ. Selective serotonin reuptake inhibitors in the treatment of affective disorders. III. Tolerability, safety and pharmacoeconomics. J Psychopharmacol 12(3 Suppl B):S55-S87, 1998.

Kaufman J, Birmaher B, Brent D, Rao U, Flynn C, Moreci P, Williamson D, Ryan N: Schedule for Affective Disorders and Schizophrenia for School-Age Children-Present and Lifetime Version (K-SADS-PL): Initial reliability and validity data. J Am Acad Child Adolesc Psychiatry 36:980-988, 1997.

Kraemer HC, Wilson GT, Fairburn CG, Agras WS: Mediators and moderators of treatment effects in randomized clinical trials. Arch Gen Psychiatry 59:877-883, 2002.

Kratochvil CJ, Newcorn JH, Arnold LE, Duesenberg D, Emslie GJ, Quintana H, Sarkis EH, Wagner KD, Gao H, Michelson D, Biederman J: Atomoxetine alone or combined with fluoxetine for treating ADHD with comorbid depressive or anxiety symptoms. J Am Acad Child Adolesc Psychiatry 44:915-924, 2005. 
Lewinsohn PM, Clarke GN: Psychosocial treatments for adolescent depression. ClinPsychol Rev 19:329-342, 1999.

MTA Cooperative Group: A 14-month randomized clinical trial of treatment strategies for attention-deficit/hyperactivity disorder. The MTA Cooperative Group. Multimodal Treatment Study of Children with ADHD. Arch Gen Psychiatry 56:1073-1086, 1999.

Mufson L, Sills R: Interpersonal Psychotherapy for depressed adolescents (IPT-A): An overview. Nord J Psychiatry 60:431-437, 2006.

Quintana H, Butterbaugh GJ, Purnell W, Layman AK: Fluoxetine monotherapy in attention-deficit/hyperactivity disorder and comorbid non-bipolar mood disorders in children and adolescents. Child Psychiatry Hum Dev 37:241-253, 2007.

Poznanski EO, Mokros HB: Children's Depression Rating ScaleRevised Manual. Los Angeles (California): Western Psychological Services, 1995.

Prince JB, Wilens TE, Biederman J, Spencer TJ, Millstein R, Polisner DA, Bostic JQ: A controlled study of nortriptyline in children and adolescents with attention deficit hyperactivity disorder. J Child Adolesc Psychopharmacol 10:193-204, 2000.

Reinecke MA, Ryan NE, Dubois DL: Cognitive-behavioral therapy of depression and depressive symptoms during adolescence: A review and meta-analysis. J Am Acad Child Adolesc Psychiatry 37:26-34, 1998.

Reynolds WM: Professional Manual for the Reynolds Adolescent Depression Scale. Odessa (Florida): Psychological Assessment Resources, 1987a.

Reynolds WM: Professional Manual for the Suicidal Ideation Questionnaire. Lutz (Florida): Psychological Assessment Resources, 1987b.

SAS Institute, SAS System for Windows, version 8.2. Cary (North Carolina): SAS Institute, 2002.
Shaffer D, Gould MS, Brasic J, Ambrosini P, Fisher P, Bird H, Aluwahlia S: A children's global assessment scale (CGAS). Arch Gen Psychiatry 40:1228-1231, 1983.

Stoll AL, Pillay SS, Diamond L, Workum SB, Cole JO: Methylphenidate augmentation of serotonin selective reuptake inhibitors: A case series. J Clin Psychiatry 57:72-76, 1996.

The Treatment for Adolescents with Depression Study (TADS) Team: Treatment for Adolescents with Depression Study (TADS): Rationale, design, and methods. J Am Acad Child Adolesc Psychiatry 42:531-542, 2003.

The Treatment for Adolescents with Depression Study (TADS) Team: Fluoxetine, cognitive-behavioral therapy, and their combination for adolescents with depression: Treatment for Adolescents with Depression Study (TADS) randomized controlled trial. JAMA 292:807-820, 2004.

The Treatment for Adolescents with Depression Study (TADS) Team: The Treatment for Adolescents with Depression Study (TADS): Demographics and clinical characteristics. J Am Acad Child Adolesc Psychiatry 44:28-40, 2005.

The Treatment for Adolescents with Depression Study (TADS) Team: The Treatment for Adolescents with Depression Study (TADS): Long-term effectiveness and safety outcomes. Arch Gen Psychiatry 64:1132-1144, 2007.

Wilens TE, Biederman J, Geist DE, Steingard R, Spencer T: Nortriptyline in the treatment of ADHD: A chart review of 58 cases. J Am Acad Child Adolesc Psychiatry 32:343-349, 1993.

Address correspondence to: Christopher J. Kratochvil, M.D. 985581 Nebraska Medical Center Omaha, NE 68198-5581

E-mail: ckratoch@unmc.edu 
